\title{
Spin relaxation in narrow wires of a two-dimensional electron gas
}

\author{
Peter Schwab, Michael Dzierzawa, and Cosimo Gorini \\ Institut für Physik, Universität Augsburg, 86135 Augsburg, Germany \\ Roberto Raimondi \\ Dipartimento di Fisica “E. Amaldi,” Università di Roma Tre, Via della Vasca Navale 84, 00146 Roma, Italy
}

(Received 14 June 2006; published 19 October 2006)

\begin{abstract}
How does an initially homogeneous spin polarization in a confined two-dimensional electron gas with Rashba spin-orbit coupling evolve in time? How does the relaxation time depend on system size? We study these questions for systems of a size that is much larger than the Fermi wavelength, but comparable and even shorter than the spin relaxation length. Depending on the confinement spin relaxation may become faster or slower than in the bulk. An initially homogeneously polarized spin system evolves into a spiral pattern.
\end{abstract}

DOI: 10.1103/PhysRevB.74.155316

PACS number(s): 72.25.Rb, 73.23.-b

\section{INTRODUCTION}

Long spin relaxation times are desirable for the operation of spintronic devices. In confined systems like quantum dots with a discrete level spectrum an initial electron spin polarization will not decay exponentially, unless the spins are coupled to extra degrees of freedom like phonons or nuclear spins. ${ }^{1,2}$ For larger or open systems, where the energy spectrum can be considered continuous, exponential decay is possible by purely elastic scattering which is then the dominant process at low enough temperatures.

In this paper we reconsider spin-relaxation in a twodimensional electron gas confined to a narrow channel, concentrating on elastic scattering processes. Our study has been motivated by a recent experiment, where the size dependence of the spin relaxation time of an $n$-InGaAs wire has been measured via Faraday rotation spectroscopy. ${ }^{3}$ The size dependence sets in when the wire width is of the order of several bulk spin relaxation lengths. The observed behavior is nonmonotonic with an initial increase followed by a sharp decrease at the smallest wire widths. The maximum spin relaxation time occurs when the width is of the order of the bulk spin relaxation length. Remarkably, while the tendency to suppress spin relaxation in confined systems has been predicted in a number of theoretical works, ${ }^{4-10}$ there has been no anticipation of the increase of the spin relaxation observed at the smallest widths. In this paper we show that spin active boundaries, not considered in the previous theoretical analysis, dramatically change the size dependence of the spin relaxation time in the small width limit and provide a useful point of view as far as the interpretation of the experiment is concerned.

The rest of the paper is organized as follows. In Sec. II we recall the method of quasiclassical Green functions and point out when a diffusion equation approach is possible. In Sec. III we consider the spin dynamics in the bulk, whereas in Sec. IV we turn to spin relaxation in finite systems. A summary is given in Sec. V.

\section{THE BASIC EQUATIONS}

We start from the Hamiltonian of a two-dimensional electron gas

$$
H=\frac{p^{2}}{2 m}+\mathbf{b} \cdot \boldsymbol{\sigma}+V(\mathbf{x})
$$

where $\mathbf{b}$ is the internal magnetic field due to the spin-orbit coupling, $\boldsymbol{\sigma}$ is the vector of Pauli matrices and $V(\mathbf{x})$ is a delta-correlated random potential due to impurity scattering. For the internal field we concentrate on the Rashba model, $\mathbf{b}=\alpha \mathbf{p} \times \mathbf{e}_{z}$, where the spin-orbit field arises due to a structural inversion asymmetry. However, our treatment can be applied also to the case of bulk inversion asymmetry, where there is in addition the Dresselhaus term ${ }^{11}$ which contributes to the two-dimensional effective Hamiltonian with $\mathbf{b}$ $=\beta\left(p_{x},-p_{y}\right)+\gamma\left(p_{x} p_{y}^{2},-p_{y} p_{x}^{2}\right)$, or to the case of a two-dimensional hole gas, where the Rashba spin-orbit field has a cubic dependence on momentum. ${ }^{12}$

To study the spin dynamics we rely on the method of the quasiclassical Green function. ${ }^{13,14}$ The latter solves the Eilenberger equation $(\hbar=1)$,

$$
\partial_{t} g+\mathbf{v}_{F} \cdot \nabla g+i[\mathbf{b} \cdot \boldsymbol{\sigma}, g]=-\frac{1}{\tau}(g-\langle g\rangle),
$$

where $\tau$ is the elastic scattering time, arising by the adoption of the standard self-consistent Born approximation for elastic impurity scattering. $\mathbf{v}_{F}$ is the Fermi velocity and $\langle\cdots\rangle$ denotes the angular average over the Fermi surface. Equation (2) is valid in the limit when both the spin-orbit energy and $\tau^{-1}$ are small compared to the Fermi energy. ${ }^{15}$ Besides the space and time dependence implied by Eq. (2), the quasiclassical Green function $g=g_{s s^{\prime}}\left(\epsilon, \hat{\mathbf{v}}_{F} ; \mathbf{x}, t\right)$ is a matrix in spin space and is a function of energy $\epsilon$ (measured with respect to the Fermi surface) and direction on the Fermi surface, $\hat{\mathbf{v}}_{F} \cdot{ }^{16}$ In the absence of external potentials and magnetic fields the particle and spin density are obtained by summing over energy, angle and spin

$$
\rho(\mathbf{x}, t)=-\frac{1}{4} N_{0} \int d \epsilon \sum_{s}\left\langle g_{s s}\left(\epsilon, \hat{\mathbf{v}}_{F} ; \mathbf{x}, t\right)\right\rangle
$$

$$
\mathbf{s}(\mathbf{x}, t)=-\frac{1}{4} N_{0} \int d \epsilon \sum_{s s^{\prime}} \frac{1}{2} \boldsymbol{\sigma}_{s s^{\prime}}\left\langle g_{s^{\prime} s}\left(\epsilon, \hat{\mathbf{v}}_{F} ; \mathbf{x}, t\right)\right\rangle
$$


When the spin-orbit energy is small compared to the scattering rate the spin-dynamics becomes diffusive in the low frequency, long wavelength limit. In this limit, all the harmonics characterizing the angle dependence of $g$ can be expressed conveniently in terms of the $s$-wave component. As a result the problem simplifies considerably and one obtains, for the angle averaged Green function, $\langle g\rangle$, a diffusion equation, which, in the presence of a general spin-orbit field, acquires the form given, for instance, in Ref. 19. For the Rashba Hamiltonian in particular the equations read

$$
\begin{gathered}
\left(\partial_{t}-D \partial_{\mathbf{x}}^{2}\right) \rho=0, \\
\left(\partial_{t}-D \partial_{\mathbf{x}}^{2}\right) s_{x}=-\frac{1}{\tau_{s}} s_{x}+2 C \partial_{x} s_{z}, \\
\left(\partial_{t}-D \partial_{\mathbf{x}}^{2}\right) s_{y}=-\frac{1}{\tau_{s}} s_{y}+2 C \partial_{y} s_{z}, \\
\left(\partial_{t}-D \partial_{\mathbf{x}}^{2}\right) s_{z}=-\frac{2}{\tau_{s}} s_{z}-2 C \partial_{x} s_{x}-2 C \partial_{y} s_{y},
\end{gathered}
$$

with $D=\frac{1}{2} v_{F}^{2} \tau, \tau_{s}=\tau /\left[2\left(\alpha p_{F} \tau\right)^{2}\right]$, and $C=v_{F} \alpha p_{F} \tau$. Since here we focus on the spin dynamics, we limit ourselves to terms to leading order in the parameter $\alpha / v_{F}$, therefore neglecting spin-charge coupling. ${ }^{15,17-19}$

\section{SPIN DYNAMICS IN THE BULK}

The spin dynamics is particularly simple for a spatially homogeneous spin density. From the diffusion equation one observes that the different spin directions decouple and the spin polarization along the $\mathbf{e}_{x, y}$ or $\mathbf{e}_{z}$ axis relaxes with the time constant $\tau_{s}$ or $\tau_{s} / 2$, respectively. This type of spin relaxation, known as the Dyakonov-Perel mechanism, ${ }^{22}$ is easily understood: the electron spin precesses around the internal field $\mathbf{b}$. Scattering from an impurity changes the direction of the internal field, and thus randomizes the spin precession. If the precession angle, $\delta \Phi \sim|\mathbf{b}| \tau$, between collisions is small, the spin dynamics resembles a diffusion. The total precession angle after $N$ scattering events is of the order $\sqrt{N} \delta \Phi$, from which the spin relaxation rate is estimated of the order $\tau_{s} \sim \tau / \delta \Phi^{2} \sim \tau /\left(\alpha p_{F} \tau\right)^{2}$.

In general we cannot rely on the diffusion equation but must solve Eq. (2). In the spatially homogeneous case the task simplifies, since again different spin directions do not couple. As a result, the spin dynamics is described by ${ }^{15}$

$$
\begin{gathered}
{\left[L\left(L^{2}+a^{2}\right)-L^{2}-\frac{1}{2} a^{2}\right] s_{x, y}=0,} \\
{\left[L\left(L^{2}+a^{2}\right)-L^{2}\right] s_{z}=0}
\end{gathered}
$$

with $L=1+\tau \partial_{t}$ and $a=2 \alpha p_{F} \tau$. Apparently the full description of the spin dynamics now requires three time constants for each component. The slowest decaying component goes as $s_{i}(t) \sim \exp \left(-\gamma_{i} t\right)$ with

$$
\gamma_{x, y}=1 / \tau_{s} \quad(a \ll 1),
$$

$$
\begin{gathered}
\gamma_{x, y}=1 / 2 \tau \quad(a \gg 1), \\
\gamma_{z}=\frac{1}{2 \tau}-\frac{1}{2 \tau} \sqrt{1-4 a^{2}},
\end{gathered}
$$

i.e., for a clean system all components decay on the time scale of the scattering time $\tau$.

For conventional diffusion the mode with the longest lifetime is homogeneous in space. Here we encounter a different situation, due to the coupling of the various spin components. From the diffusion equation, for instance, the modes with the longest lifetime are found to form an elliptically modulated spin spiral,

$$
\mathbf{s}(\mathbf{x}, t) \propto\left[\mathbf{e}_{q} \cos (\mathbf{q} \cdot \mathbf{x})+A \mathbf{e}_{z} \sin (\mathbf{q} \cdot \mathbf{x})\right] \exp \left(-\gamma_{0} t\right),
$$

where the vector $\mathbf{q}$ lives in the $x-y$ plane with $D q^{2}=15$ / $16 \tau_{s}, A=3 / \sqrt{15}$, and $\gamma_{0}=7 / 16 \tau_{s}$. These long-living modes have been investigated in Refs. 20 and 21.

\section{SPIN DYNAMICS IN FINITE SYSTEMS}

For finite systems the equations must be supplemented by boundary conditions. ${ }^{23-25}$ For specular scattering where an in-going trajectory is scattered into one outgoing direction the boundary condition for the Eilenberger equation reads ${ }^{25}$

$$
g_{s s^{\prime}}^{\text {out }}=\sum_{s_{1} s_{1}^{\prime}} S_{s s_{1}} g_{s_{1} s_{1}^{\prime}}^{\text {in }} S_{s_{1}^{\prime} s^{\prime}}^{+}
$$

The unitary matrix $S$ describes the surface scattering. By decomposing the Green function in charge and spin components, $g_{s s^{\prime}}=g_{0} \delta_{s s^{\prime}}+\mathbf{g} \cdot \boldsymbol{\sigma}_{s s^{\prime}}$, the boundary condition is equivalently expressed as

$$
g_{0}^{\text {out }}=g_{0}^{\text {in }}, \quad \mathbf{g}^{\text {out }}=R \mathbf{g}^{\text {in }}
$$

with an orthogonal matrix $R$ that rotates the spin at the surface. Charge conservation implies that no current flows through the boundary,

$$
\left\langle\mathbf{n} \cdot \mathbf{v}_{F} g_{0}\right\rangle \propto \mathbf{n} \cdot \mathbf{j}_{c}=0,
$$

where $\mathbf{n}$ is a vector normal to the boundary. For a spin conserving boundary $(R=1)$ also all components $\alpha$ of the spin current perpendicular to the surface are zero,

$$
\left\langle\mathbf{n} \cdot \mathbf{v}_{F} g_{\alpha}\right\rangle \propto \mathbf{n} \cdot \mathbf{j}_{\alpha}=0 .
$$

For the general case, $R \neq 1$, Eq. (18) is not valid.

In the following we consider two types of boundary conditions. First we consider a spin-conserving boundary, where

$$
\left|\mathbf{k}_{\text {in }} s\right\rangle \rightarrow\left|\mathbf{k}_{\text {out }} s\right\rangle,
$$

such that $S$ and $R$ are unit matrices. As a second example we will consider a boundary that scatters adiabatically, i.e., an incoming wave in an eigenstate $\left|\mathbf{k}_{\text {in }} \pm\right\rangle$ of the Hamiltonian (1) —not including disorder-is scattered into the same band,

$$
\left|\mathbf{k}_{\text {in }} \pm\right\rangle \rightarrow\left|\mathbf{k}_{\text {out }} \pm\right\rangle,
$$

as it is expected for a smooth confining potential. ${ }^{26-28}$ Since the eigenstates in the presence of the field $\mathbf{b}$ are spin polar- 
ized parallel to $\mathbf{b}$, the adiabatic boundary implies a rotation of the spin polarization, $S=e^{i \sigma_{z} \varphi}$, where $\varphi$ is the angle between the electron momentum and the normal of the interface.

Before presenting our numerical results for the spin relaxation obtained by solving Eq. (2), it is useful to derive the boundary conditions in the diffusive regime. The idea is to solve the Eilenberger equation near the boundary assuming that the angular average of $g$ varies only slowly on the scale of the mean free path, $l$. This is justified when the spin relaxation length $L_{s}=\sqrt{D \tau_{s}}$ is much longer than $l$. For directions pointing into the boundary one can then use the expansion valid in the bulk,

$$
\mathbf{g}^{\text {in }}=\langle\mathbf{g}\rangle-\tau \mathbf{v}_{F} \cdot \nabla\langle\mathbf{g}\rangle+2 \tau \mathbf{b} \times\langle\mathbf{g}\rangle .
$$

Combining (16) and (21) yields a relation that connects linearly the three spin components of the angular averaged Green function, $\langle\mathbf{g}\rangle$, and its spatial derivatives. The resulting form of the boundary condition depends on the rotation matrix $R(\varphi)$,

$$
\int_{\varphi_{\text {in }}} \frac{\mathrm{d} \varphi}{2 \pi}(1+R)\left[\langle\mathbf{g}\rangle-\tau \mathbf{v}_{F} \cdot \nabla\langle\mathbf{g}\rangle+2 \pi \mathbf{b} \times\langle\mathbf{g}\rangle\right]=\langle\mathbf{g}\rangle .
$$

For the Rashba model, using the expression for the charge and the spin current in the diffusive limit, we get for spinconserving boundary conditions, 5,29

$$
\begin{gathered}
-D \partial_{x} s_{x}-C s_{z}=\mathbf{n} \cdot \mathbf{j}_{x}=0, \\
-D \partial_{x} s_{y}=\mathbf{n} \cdot \mathbf{j}_{y}=0, \\
-D \partial_{x} s_{z}+C s_{x}=\mathbf{n} \cdot \mathbf{j}_{z}=0,
\end{gathered}
$$

where $\mathbf{n}$ is in the $x$ direction. For adiabatic boundary conditions, in contrast, angular averaging of Eq. (22) yields that

$$
s_{x}=0 \text { and } s_{y}=0 \text {, }
$$

while the $z$ component of the spin is still conserved and therefore Eq. (25) remains valid.

Figure 1 shows the time evolution of the spin profile for a long wire of width $L=200 l$, where $l=v_{F} \tau$ is the elastic mean free path. Initially the spin was homogeneously polarized in the $z$ direction. The results were obtained from the Eilenberger equation with $\alpha p_{F} \tau=0.1$, and the conserving boundary condition, $S_{s s^{\prime}}=\delta_{s s^{\prime}}$. Inside the wire one observes a homogeneous decay of the spin polarization, with the time constant $\tau_{s} / 2$. At the boundaries long living modes show up which dominate the spin profile in the long time limit.

For further investigation of these modes we write the spin diffusion equation, Eqs. (6)-(8), as $\partial_{t} \mathbf{s}+\hat{\gamma} \mathbf{s}=0$, and determine the eigenvalues and eigenmodes of the operator $\hat{\gamma}$. The eigenmodes are superpositions of plane waves. The low frequency spectrum of $\hat{\gamma}$ is shown in Fig. 2 as a function of the wire width. Recall that the smallest eigenvalue for a bulk system is $\gamma_{0}=7 / 16 \tau_{s}$. The modes with smaller decay rate have a complex wave vector and are thus localized near the edges of the wire. For a wide system we find a continuum

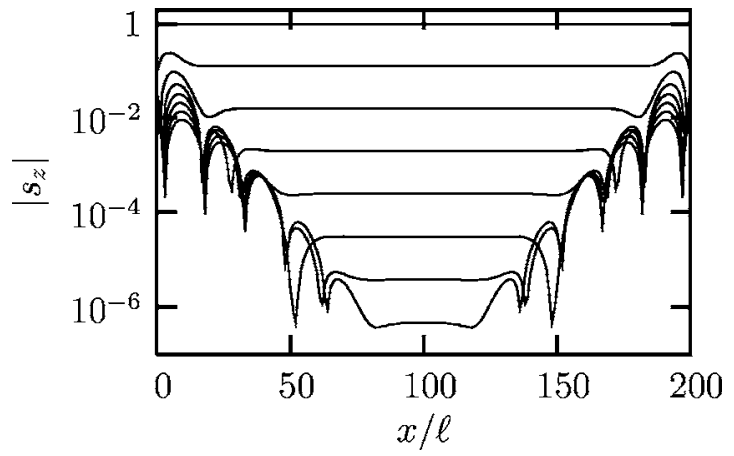

FIG. 1. Time evolution of the spin polarization in a wide channel $\left(L=200 l \approx 40 L_{s}, \alpha p_{F} \tau=0.1\right)$ with conserving boundary conditions. The curves from top to bottom correspond to different times, with $\Delta t=50 \tau \approx \tau_{s}$. $s_{z}$ changes sign at various positions where a steep drop of $\left|s_{z}\right|$ is visible in the figure.

of eigenvalues above $\gamma_{0}$, and two localized modes at $\gamma$ $\approx 0.382 / \tau_{s}$.

For a narrow wire most strikingly one eigenvalue goes to zero with decreasing width, asymptotically as

$$
\gamma \tau_{s} \simeq \frac{1}{12}\left(\frac{L}{L_{s}}\right)^{2}
$$

This corresponds to the suppression of spin relaxation in small systems reported earlier by other authors. ${ }^{5-10}$ This effect can be traced back to the specific form of the spin-orbit field in the Rashba Hamiltonian - being proportional to the velocity. ${ }^{7}$ Here we formulate the argument for a system including Rashba and also linear Dresselhaus term within the spin-diffusion equation approach. For a spin profile that is homogeneous in the $y$ direction the angular averaged Eilenberger equation, Eq. (2), yields

$$
\partial_{t} s_{x}+\partial_{x} j_{x}^{x}=-2 m \alpha j_{z}^{x}-2 m \beta j_{z}^{y}
$$

$$
\partial_{t} s_{y}+\partial_{x} j_{y}^{x}=-2 m \alpha j_{z}^{y}-2 m \beta j_{z}^{x}
$$

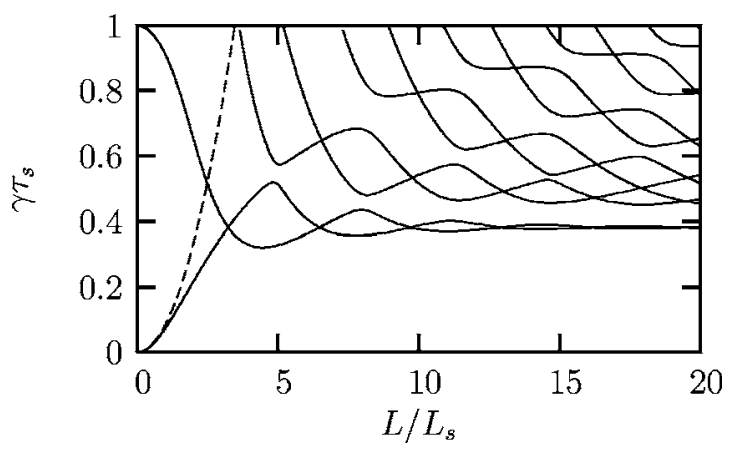

FIG. 2. Lowest eigenvalues of the spin-diffusion operator for the Rashba model and conserving boundary conditions, Eqs. (23)-(25). Modes with $\gamma<7 / 16 \tau_{s}$ have a complex wave vector, and can therefore exist only at the edges of the wire. The dashed curve is $\gamma \tau_{s}$ $=\left(L / L_{s}\right)^{2} / 12$ obtained in Ref. 5 for very narrow wires. 


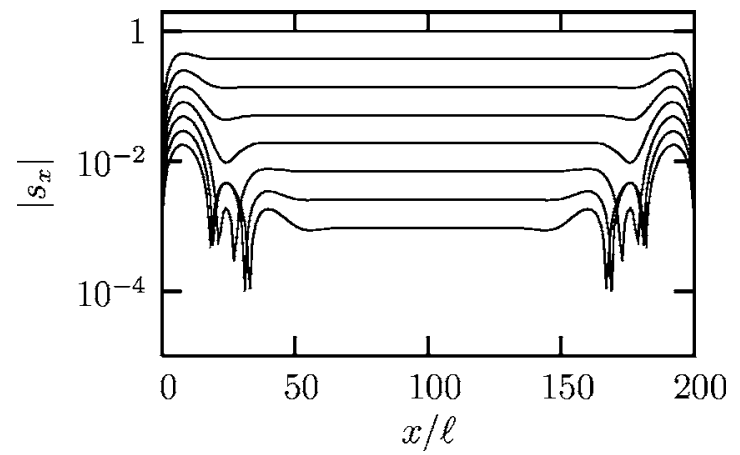

FIG. 3. Time evolution of the spin polarization in the $x$ direction for a wire with adiabatic boundary condition for the same set of parameters as in Fig. 1.

$$
\partial_{t} s_{z}+\partial_{x} j_{z}^{x}=2 m \alpha\left(j_{x}^{x}+j_{y}^{y}\right)+2 m \beta\left(j_{x}^{y}+j_{y}^{x}\right),
$$

where $j_{\alpha}^{\beta}$ is the spin current polarized in the $\alpha$ and flowing in the $\beta$ direction. In the diffusive limit the spin current densities are given by

$$
j_{\alpha}^{\beta}=-D \partial_{\beta} s_{\alpha}+2 \tau\left\langle v_{F}^{\beta}(\mathbf{b} \times \mathbf{s})_{\alpha}\right\rangle,
$$

which allows to reproduce the spin-diffusion equation, Eqs. (6)-(8). In narrow systems the slow modes have a smooth density profile, such that to leading order in the system size the current can be considered constant in space. For a quantum dot, i.e., a system that is confined in all spatial directions, the vanishing of the spin current through the boundaries then implies immediately that $\partial_{t} \mathbf{s}=0$. For a narrow wire the situation becomes slightly more complicated since only currents flowing into the boundary are zero, which after some algebra leads to

$$
\partial_{t} \mathbf{s}=-\frac{1}{\tau_{s}} \frac{1}{\alpha^{2}}\left(\begin{array}{ccc}
\beta^{2} & \alpha \beta & 0 \\
\alpha \beta & \alpha^{2} & 0 \\
0 & 0 & \alpha^{2}+\beta^{2}
\end{array}\right)\left(\begin{array}{c}
s_{x} \\
s_{y} \\
s_{z}
\end{array}\right) .
$$

In the absence of the Dresselhaus term $(\beta=0)$ this means that $\partial_{t} s_{x}=0$, and $\partial_{t} s_{y}=-s_{y} / \tau_{s}, \partial_{t} s_{z}=-s_{z} / \tau_{s}$, which implies that the long-living mode in Fig. 2 is polarized in the $x$ direction. In the presence of both a Rashba and a Dresselhaus term, the spin is still conserved for one direction which depends on the relative strength of the two terms: Perpendicular to the boundary when the Rashba term dominates, parallel to the boundary when the Dresselhaus term is larger, and somewhere in between (but always in-plane) when both terms are comparable in size.

The above results change considerably when different boundary conditions are applied. Figure 3 shows the time evolution of a spin polarization using adiabatic boundary conditions, Eq. (26). Here the spin has been prepared in the $x$ direction, i.e., perpendicular to the boundary. In this case the boundary mode is absent, and the asymptotic decay of the spin polarization is ruled by an inhomogeneous, but extended mode. The spectrum of the spin diffusion operator is shown in Fig. 4. The boundary condition implies that the eigenmodes are $s_{x, y} \propto \sin (q x), s_{z} \propto \cos (q x)$ with $q=n \pi / L$; the eigenvalues are given by

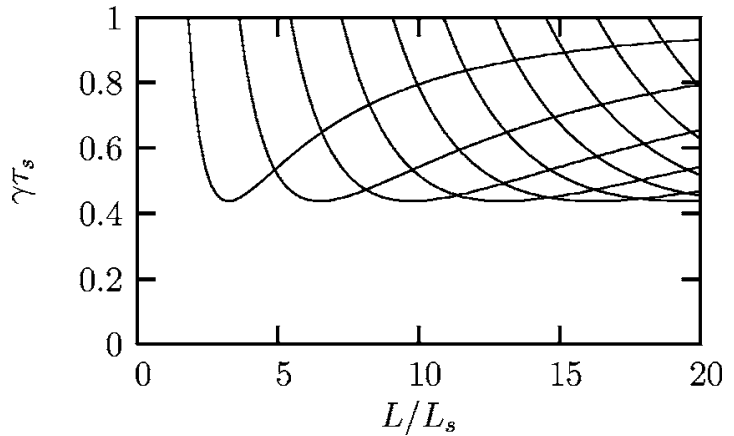

FIG. 4. Lowest eigenvalues of the spin-diffusion operator for adiabatic boundary conditions.

$$
\gamma(q)=D q^{2}+\frac{3}{2 \tau_{s}} \pm \frac{1}{2 \tau_{s}} \sqrt{1+16 L_{s}^{2} q^{2}} .
$$

By inserting the allowed $q$ values the spectrum shown in Fig. 4 is reproduced. In contrast to the previous case of a spinconserving boundary, here all the diffusion modes show an increasing spin relaxation rate at the smallest wire widths. In particular, all the modes show a nonmonotonous behavior as a function of the wire width with a minimum at $L / L_{s}$ $=(4 \pi / \sqrt{15}) n \approx 3 n$, where $n$ is the mode index. For width $L$ $<L_{s}$ all spin-diffusion modes relax fast.

\section{SUMMARY}

Motivated by a recent experiment ${ }^{3}$ we studied spin relaxation in narrow wires with spin-orbit coupling. To this end we have solved the Eilenberger equation and the spin diffusion equation in the presence of spin-orbit interaction ${ }^{15}$ supplemented by boundary conditions for both charge and spin degrees of freedom assuming translational invariance along the wire.

The spin-diffusion equation has extra, off-diagonal gradient terms that couple the different spin directions. Due to these terms there exist inhomogeneous spin profiles, typically spin spirals, that decay slower than a homogeneous spin configuration and that show up close to the edges of the wire. Therefore there is a tendency for the spin relaxation to slow down, the stronger the influence of the boundary becomes, i.e., when decreasing the width of the wire.

For very narrow wires, i.e., narrower than the spin relaxation length, the spin relaxation rate depends crucially on the boundary condition and on the form of the spin-orbit coupling. For models with linear-in-momentum spin-orbit field and spin-conserving boundaries the spin relaxation rate goes to zero with the system size. It is interesting to note that while in a quantum dot all spin components are conserved in the finite size limit, in the case of a narrow wire only one of them is conserved. The direction of this conserved component depends on the relative strength of the Rashba and Dresselhaus terms.

On the other hand, in the case of spin-active boundaries, the spin is not conserved and the relaxation rate grows when the wire becomes very narrow. Whereas the spin-relaxation in the bulk is a slow process governed by spin precession, the relaxation at the boundary is induced by instantaneous 
spin-flip events as in the Elliott-Yafet mechanism. We believe that the experimentally observed ${ }^{3}$ sharp increase of the spin relaxation rate in very narrow wires can be explained by spin scattering at the boundary.

\section{ACKNOWLEDGMENT}

This work was supported by the Deutsche Forschungsgemeinschaft through Sonderforschungsbereich 484.
${ }^{1}$ A. V. Khaetskii and Y. V. Nazarov, Phys. Rev. B 61, 12639 (2000).

${ }^{2}$ I. A. Merkulov, A. L. Efros, and M. Rosen, Phys. Rev. B 65 , 205309 (2002).

${ }^{3}$ A. W. Holleitner, V. Sih, R. C. Myers, A. C. Gossard, and D. D. Awschalom, Phys. Rev. Lett. 97, 036805 (2006).

${ }^{4}$ A. Bournel, D. Dollfus, P. Bruno, and P. Hesto, Eur. Phys. J.: Appl. Phys. 4, 1 (1998).

${ }^{5}$ A. G. Mal'shukov and K. A. Chao, Phys. Rev. B 61, R2413 (2000).

${ }^{6}$ A. A. Kiselev and K. W. Kim, Phys. Rev. B 61, 13115 (2000).

${ }^{7}$ B. I. Halperin, A. Stern, Y. Oreg, J. N. H. J. Cremers, J. A. Folk, and C. M. Marcus, Phys. Rev. Lett. 86, 2106 (2001).

${ }^{8}$ I. L. Aleiner and V. I. Fal'ko, Phys. Rev. Lett. 87, 256801 (2001).

${ }^{9}$ C.-H. Chang, A. G. Mal'shukov, and K. A. Chao, Phys. Rev. B 70, 245309 (2004).

${ }^{10}$ O. Zaitsev, D. Frustaglia, and K. Richter, Phys. Rev. Lett. 94, 026809 (2005).

${ }^{11}$ G. Dresselhaus, Phys. Rev. 100, 580 (1955).

${ }^{12}$ R. Winkler, Phys. Rev. B 62, 4245 (2000); J. Schliemann and D. Loss, ibid. 71, 085308 (2005).

${ }^{13}$ J. Rammer and H. Smith, Rev. Mod. Phys. 58, 323 (1986).

${ }^{14}$ P. Schwab and R. Raimondi, Ann. Phys. 12, 471 (2003).

${ }^{15}$ R. Raimondi, C. Gorini, P. Schwab, and M. Dzierzawa, Phys. Rev. B 74, 035340 (2006).
${ }^{16}$ More precisely, in a nonequilibrium Green function formalism, $g$ is the so-called Keldysh component and may be seen as the quantum analog of the Boltzmann distribution function.

${ }^{17}$ A. A. Burkov, A. S. Nunez, and A. H. MacDonald, Phys. Rev. B 70, 155308 (2004).

${ }^{18}$ E. G. Mishchenko, A. V. Shytov, and B. I. Halperin, Phys. Rev. Lett. 93, 226602 (2004).

${ }^{19}$ A. G. Mal'shukov, L. Y. Wang, C. S. Chu, and K. A. Chao, Phys. Rev. Lett. 95, 146601 (2005).

${ }^{20}$ V. A. Froltsov, Phys. Rev. B 64, 045311 (2001).

${ }^{21}$ Y. V. Pershin, Phys. Rev. B 71, 155317 (2005).

${ }^{22}$ M. I. Dyakonov and V. I. Perel, Phys. Lett. 35A, 459 (1971).

${ }^{23}$ A. V. Zaitsev, Zh. Eksp. Teor. Fiz. 86, 1742 (1984) [Sov. Phys. JETP 59, 1015 (1984)].

${ }^{24}$ A. L. Shelankov, J. Low Temp. Phys. 60, 29 (1985).

${ }^{25}$ A. Millis, D. Rainer, and J. A. Sauls, Phys. Rev. B 38, 4504 (1988).

${ }^{26}$ A. O. Govorov, A. V. Kalameitsev, and J. P. Dulka, Phys. Rev. B 70, 245310 (2004).

${ }^{27}$ P. G. Silvestrov and E. G. Mishchenko, cond-mat/0506516 (unpublished).

${ }^{28}$ H. Chen, J. J. Heremans, J. A. Peters, A. O. Govorov, N. Goel, S. J. Chung, and M. B. Santos, Appl. Phys. Lett. 86, 032113 (2005).

${ }^{29}$ V. M. Galitski, A. A. Burkov, and S. Das Sarma, Phys. Rev. B 74, 115331 (2006). 